\title{
A CLASS OF SIMPLE LATTICE-ORDERED GROUPS
}

\section{CHARLES HOLLAND ${ }^{1}$}

A lattice-ordered group ( $l$-group) is said to be regular if no positive element of the group is disjoint from any of its conjugates. It is well known that every simple regular $l$-group is totally ordered [5]. The subgroups of the reals are the most elementary examples of regular simple $l$-groups; other examples can be found in [2] and [6]. In this note we investigate a class of simple $l$-groups at the opposite extreme from the regular ones. We are concerned with $l$-groups which contain an insular (defined below) element. An insular element is, roughly speaking, an element which is strongly disjoint from one of its conjugates. In [4] it was shown that every $l$-group can be represented as an $l$-group of automorphisms of a totally ordered set, and it was shown that the $l$-group of automorphisms of the real line with bounded support is simple. It is natural to ask which simple $l$-groups can be represented as automorphisms of an ordered set with bounded support. Our main result is that these are exactly the simple $l$-groups containing an insular element. We also construct several examples of such groups.

If $L$ is a totally ordered set and $f$ is an order-preserving permutation of $L$, we call $f$ an automorphism of $L$. The support of $f$ consists of those $x \in L$ such that $x f \neq x$. An automorphism of $L$ is bounded if its support lies in a closed interval of $L$. An l-group of automorphisms of $L$ is a group of automorphisms of $L$ (under composition) which is a lattice under the operations $\cap$ and $U$ defined by $x(f \cap g)=(x f) \cap(x g)$ and dually. Such a group is a lattice-ordered group in the usual sense [1]. If $G$ is an $l$-group of automorphisms of $L, G$ is o-primitive on $L$ if there is no equivalence relation $E$ on $L$ such that (1) $E$ is a congruence; that is, for all $x, y \in L, f \in G, x E y$ implies $x f E y f$, and (2) $E$ is convex; that is, each $E$-class is a convex subset of $L$. For elements $f, g \geqq 1$ of an $l$-group $G, f$ is right of $g$ if for all $1 \leqq h \in G, g \cap h^{-1} f h=1$. An element $g \in G$ is insular if for some conjugate $g^{*}$ of $g, g^{*}$ is right of $g$.

LEMMA. Let $G$ be a transitive l-group of automorphisms of an ordered set $L$. An element $1<g \in G$ is insular if and only if $g$ is bounded.

Received by the editors September 13, 1963.

1 This research was supported by a grant from the National Science Foundation. 
Proof. Suppose the support of $g$ lies in the closed interval $[a, b]$ of $L$. By transitivity, there exists $1 \leqq h \in G$ such that $a h=b$. Let $g^{*}=h^{-1} g h$. Then for every $x \in[a, b]$, and for every $1 \leqq k \in G, x k^{-1} h^{-1}$ $\leqq x h^{-1} \leqq b h^{-1}=a$. Hence $x k^{-1} h^{-1} g=x k^{-1} h^{-1}$. Thus $x k^{-1} g^{*} k=x$. Therefore the support of $k^{-1} g^{*} k$ lies outside the interval $[a, b]$, and so $g \cap k^{-1} g^{*} k=1$.

Conversely, suppose $g^{*}=k^{-1} g k$ is right of $g$. Without loss of generality, $1 \leqq k$. There exists $x \in L$ such that $x<x g^{*}$. If there exists $y \in L$ such that $x<y<y g$, then by transitivity there exists $1<f \in G$ such that $x f=y$, and thus $y f^{-1} g^{*} f=x g^{*} f>x f=y$, which implies $y\left(g \cap f^{-1} g^{*} f\right)$ $>y$; that is, $g \cap f^{-1} g^{*} f>1$, a contradiction. Hence the support of $g$ is bounded above by $x$. In a similar manner, there exists $z \in L$ such that $z<z g$. Let $w \leqq z k^{-1}$. Then $w k \leqq z$, so there exists $h \in G$ such that $1 \leqq h$ and $w k h=z$. Since $g \cap h^{-1} g^{*} h=1, \quad z=z h^{-1} k^{-1} g k h=w g k h \geqq w k h=z$. Hence $w g k h=w k h$, and $w g=w$. Therefore, the support of $g$ is bounded below by $z k^{-1}$.

TheOREM. $G$ is a simple l-group containing an insular element if and only if $G$ is a transitive o-primitive l-group of bounded automorphisms of a totally ordered set.

Proof. Let $G$ be a simple $l$-group containing an insular element $g$. Every simple $l$-group is a transitive $l$-group of automorphisms of an ordered set [4, Theorem 3, Corollary 2]. By the lemma, $g$ must be bounded. It is easily seen that the bounded elements of $G$ form an $l$-ideal. Thus every element of $G$ is bounded. Hence $G$ is a transitive $l$-group of bounded automorphisms of an ordered set $L$. Of course, $G$ need not be $o$-primitive on $L$. Let the support of $1 \neq f \in G$ lie in the closed interval $[a, b]$ of $L$. If $E$ is any convex congruence on $L$, then

$$
G_{E}=\{g \in G \mid x E(x g) \text { for all } x \in L\}
$$

is an $l$-ideal of $G$. Hence, for no proper convex congruence $E$ is $a E b$, since otherwise, $f \in G_{E}$ and hence $G=G_{E}$, which contradicts the transitivity of $G$. It follows that the union of any tower of proper convex congruences on $L$ is a proper convex congruence. By Zorn's lemma, there is a maximal proper convex congruence $M$ on $L$. The natural mapping induces a total order on $L^{\prime}=L / M$. For $x M \in L^{\prime}$ and $g \in G$, define $(x M) g=(x g) M$. Then $G$ is a transitive $o$-primitive $l$-group of bounded automorphisms of $L^{\prime}$.

Conversely, let $G$ be a transitive $o$-primitive $l$-group of bounded automorphisms of an ordered set $L$. Let $\{1\} \neq N$ be an $l$-ideal of $G$. Define an equivalence relation $E$ on $L$ by: $x E y$ if and only if there exists $1<f \in N$ such that $x \leqq y f$ and $y \leqq x f$. Then it is easily verified 
that $E$ is a convex congruence. Since $N \neq\{1\}$, and for any $f \in N$, $x E(x f)$ for all $x \in L$, at least one $E$-class contains more than one point. Therefore, since $G$ is $o$-primitive, there is just one $E$-class. Now let $1<g \in G$. By assumption, the support of $g$ lies in some interval $[a, b]$. Since $a E b$, there exists $1<f \in N$ such that $b \leqq a f$. Hence, for any $x \in[a, b], x g \leqq b \leqq a f \leqq x f$. For any $x \in L \backslash[a, b], x g=x \leqq x f$. Thus, $g \leqq f$, and as $N$ is convex, $g \in N$. Therefore, $G=N$, and $G$ is simple. Finally, by the lemma, every positive element of $G$ is insular. This completes the proof of the theorem.

CoRollary 1. If $G$ is a simple l-group with an insular element, then every positive element of $G$ is insular.

CoROLlary 2. If $G$ is a simple l-group with an insular element, then for every $1<g \in G$ there is an infinite collection of pairwise disjoint conjuga'es of $g$.

It is possible that the conclusion of Corollary 2 would follow from the weaker hypothesis that $G$ be simple and not totally ordered. It can be shown, using results in [3] that any simple nontotally ordered $l$-group contains an infinite collection of pairwise disjoint elements.

We close this note with some examples. Let $G$ be the $l$-group of all bounded automorphisms of the ordered set $L$. If $G$ is o-doubly transitive in the sense that for any $a, b, c, d \in L$ with $a<b$ and $c<d$, there exists $g \in G$ such that $a g=c$ and $b g=d$, then clearly $G$ is $o$-primitive on $L$. The following is also useful: If $G$ is transitive on $L$ and $L$ is relatively complete, then $G$ is o-primitive on $L$. For if $E$ were a nontrivial convex congruence on $L$, then there would be some nontrivial $E$-class containing an end point; but then $G$ could not be transitive on $L$.

In particular, if $L$ is an ordered field, then the group of automorphisms of $L$ is $o$-doubly transitive, and hence the $l$-group $G$ of bounded automorphisms of $L$ is simple. $G$ is also simple if $L$ is the long line, the inverted long line, or the double long line. A somewhat different example in which $L$ is relatively complete and yet not locally isomorphic to the reals arises in the following way. Consider the field $F$ of semi-infinite polynomials of the form $\sum_{i=-\infty}^{n} r_{i} x^{i}$ with integer exponents and real coefficients, ordered lexicographically from the largest exponent. Then the $l$-group of automorphisms of $F$ is 0 -doubly transitive. It follows that if $a_{1}<a_{2}<\cdots$ and $b_{1}<b_{2}<\cdots$ are bounded countable sequences of elements of $F$, then there is an automorphism of $F$ which maps $a_{i}$ onto $b_{i}$ for each $i$. A similar statement holds for decreasing sequences. Now let $L$ be the completion of $F$ by Dedekind cuts. Every automorphism of $F$ can be extended uniquely 
to an automorphism of $L$. Moreover, every element $a \in L$ is the limit of two sequences $\left\{a_{i}\right\}$ and $\left\{b_{i}\right\}$ of elements of $F$ such that

$$
a_{1}<a_{2}<\cdots<a<\cdots<b_{2}<b_{1} \text {. }
$$

From this, it follows that the $l$-group $G$ of bounded automorphisms of $L$ is transitive on $L$. Hence $G$ is simple.

Finally, we give an example of a nontotally ordered simple $l$-group which does not contain an insular element. Let $t$ be that automorphism of the real line $R$ defined by $x t=x+1$. Let $G$ consist of all those automorphisms $f$ of $R$ such that $t f=f t$ ( $f$ is "periodic"). Then $G$ is a transitive sub-l-group of the $l$-group of all automorphisms of $R$. To show that $G$ is simple, let $g$ and $f$ be positive elements of $G$. Then the support of $g$ meets the interval $[0,1]$, where 0 and 1 denote real numbers throughout the following argument. Hence for a finite number of conjugates $g_{1}, g_{2}, \cdots, g_{n}$ of $g$, the support of $g^{*}=g_{1}$ $\cup g_{2} \cup \ldots \cup g_{n}$ contains $[0,1]$. Thus by periodicity $g^{*}$ has no fixed points in $R$. It follows that for some positive integer $m, 1 f<O\left(g^{*}\right)^{m}$. Therefore, for all $x \in[0,1]$,

$$
x f \leqq 1 f<O\left(g^{*}\right)^{m} \leqq x\left(g^{*}\right)^{m} .
$$

By periodicity again, $y f<y\left(g^{*}\right)^{m}$ for all $y \in R$. That is, $f<\left(g^{*}\right)^{m}$. Thus any $l$-ideal of $G$ containing $g$ also contains $f$. Therefore, $G$ is simple. Clearly, $G$ does not contain an element of bounded support, and therefore, no element of $G$ is insular.

\section{REFERENCES}

1. G. Birkhoff, Lattice theory, rev. ed., Amer. Math. Soc. Colloq. Publ. Vol. 25, Amer. Math. Soc., Providence, R. I., 1948.

2. A. H. Clifford, $A$ noncommutative ordinally simple linearly ordered group, Proc. Amer. Math. Soc. 2 (1952), 902-903.

3. P. Conrad, The structure of a lattice-ordered group with a finite number of elements, Michigan Math. J. 7 (1960), 171-180.

4. C. Holland, The l-group of automorphisms of an ordered set, Michigan Math. J. 10 (1963), 399-408.

5. P. Lorenzen, Über halbgeordnete Gruppen, Math. Z. 52 (1949), 484-526.

6. B. H. Neumann, On ordered groups, Amer. J. Math. 71 (1948), 1-18.

University of Chicago 